\title{
The Term Structure of Interest Rates under Regime Shifts and Jumps
}

\author{
Shu Wu and Yong Zeng*
}

September 2005

\begin{abstract}
This paper develops a tractable dynamic term structure models under jump-diffusion and regime shifts with time varying transition probabilities. The model allows for regime-dependent jumps while both jump risk and regime-switching risk are priced. Closed form solution for the term structure is obtained for an affine-type model under loglinear approximation.
\end{abstract}

JEL Classification: G12, E43, E52

Key Words: Term Structure, Regime Switching, Jump Diffusion, Marked Point Process

${ }^{*}$ Shu $\mathrm{Wu}$ is with Department of Economics at the University of Kansas, Summerfield Hall 213, Lawrence, KS 66045. Tel: (785) 864 2868, email: shuwu@ku.edu. Yong Zeng is with Department of Mathematics and Statistics at University of Missouri at Kansas City, Tel: (816) 235 5850, email: zeng@mendota.umkc.edu. 


\section{Introduction}

The Fed conducts monetary policy by targeting the short-term interest rate. Many dynamic models of the term structure of interest rates have included a Poisson jump component to reflect the impact of the policy actions. ${ }^{1}$ A notable feature of the monetary policy behavior is that those discrete changes in the interest rate target of the same direction are very persistent. For example, the Fed decreased the interest rate target 12 times consecutively between January 2001 and November 2002, and since June 2003 there have been 11 interest rate hikes by the Fed without a single decrease. Presumably such shifts in the overall monetary policy stance (from accommodative to tightening or vice versa) have more important effects on interest rates than a single interest rate change does.

In this paper we propose a regime-dependent jump-diffusion model of the term structure of interest rates to capture the effects of not only discrete jumps in the interest rate target, but also shifts in the policy regime. The paper is based on a strand of recent studies on term structure models with regime shifts, including Landen (200), Bansal and Zhou (2002), Dai, Singleton and Yang (2003) and $\mathrm{Wu}$ and Zeng (2005) among others. The main contribution of the present paper is that it proposes a simple framework to model both discrete jumps and regime shifts in interest rates.

\section{The Model}

\section{$2.1 \quad$ State variables}

We assume that the first $L$ state variables, $X_{t}$, are described by the following equation

$$
d X_{t}=\Theta\left(X_{t-}, S_{t-}\right) d t+\Sigma\left(X_{t-}, S_{t-}\right) d W_{t}+J\left(X_{t-}, S_{t-}\right) d N_{t}
$$

where $S_{t}$ is another state variable following a $K$-regime continuous-time Markov chain to be specified below; $X_{t}$ and $\Theta\left(X_{t-}, S_{t-}\right)$ are both $L \times 1$ vectors; $\Sigma\left(X_{t-}, S_{t-}\right)$ is a $L \times L$ matrix; $W_{t}$ is a $L \times 1$ vector of independent standard Brownian motions; $N_{t}$ is a $L \times 1$ vector of independent

\footnotetext{
${ }^{1}$ Some recent studies include Ahn and Thompson (1998), Das (2002), Piazzesi (2005) among others.
} 
Poisson processes with $L \times 1$ time-varying and regime-dependent intensity $\delta_{J}\left(X_{t-}, S_{t-}\right) ; J\left(X_{t-}, S_{t-}\right)$ is a $L \times L$ matrix of regime-dependent random jump size with a conditional density $g\left(J \mid X_{t-}, S_{t-}\right)$. Given $\left\{X_{t-}, S_{t-}\right\}$, we assume that $J\left(X_{t-}, S_{t-}\right)$ is serially independent and is also independent of $W_{t}$ and $N_{t}$.

To get a convenient representation of $S_{t}$, we define the mark space $E$ as

$$
E=\{(i, j): i \in\{1, \ldots, K\}, j \in\{1,2, \ldots, K\}, i \neq j\}
$$

with $\sigma$-algebra $\mathcal{E}=2^{E}$. Let $z=(i, j)$ be a generic point in $E$ and $A$ a subset of $E$. A marked point process, $\mu(t, A)$, counts the cumulative number of regime shifts that belong to $A$ during $(0, t] . \mu(t, \cdot)$ can be uniquely characterized by its stochastic intensity kernel, ${ }^{2}$, which is assumed to be

$$
\gamma_{\mu}(d t, d z)=h\left(z, X_{t-}\right) \mathbf{I}\left\{S_{t-}=i\right\} \epsilon_{z}(d z) d t
$$

where $h\left(z, X_{t-}\right)$ is the regime-shift (from regime $i$ to $j$ ) intensity at $z=(i, j)$, $\mathbf{I}\left\{S_{t-}=i\right\}$ is an indicator function, and $\epsilon_{z}(A)$ is the Dirac measure (on a subset $A$ of $E$ ) at point $z$ (defined by $\epsilon_{z}(A)=1$ if $z \in A$ and 0 , otherwise). Heuristically, for $z=(i, j), \gamma_{\mu}(d t, d z)$ is the conditional probability of a shift from regime $i$ to regime $j$ during $[t, t+d t)$ given $X_{t-}$ and $S_{t-}=i$. The compensator of $\mu(t, A)$ is then given by

$$
\gamma_{\mu}(t, A)=\int_{0}^{t} \int_{A} h\left(z, X_{\tau-}\right) \mathbf{I}\left\{S_{\tau-}=i\right\} \epsilon_{z}(d z) d \tau .
$$

This simply implies that $\mu(t, A)-\gamma_{\mu}(t, A)$ is a martingale.

Using the above notations, the evolution of the regime $S_{t}$ can be conveniently represented as

$$
d S_{t}=\int_{E} \zeta(z) \mu(d t, d z)
$$

with its compensator given by

$$
\gamma_{S}(t) d t=\int_{E} \zeta(z) \gamma_{\mu}(d t, d z)
$$

where $\zeta(z)=\zeta((i, j))=j-i$.

\footnotetext{
${ }^{2}$ See Last and Brandt (1995) for detailed discussion of marked point process, stochastic intensity kernel and related results.
} 
Without loss of generality, we assume that the instantaneous short-term interest $r_{t}$ is a linear function of the state variables $X_{t}$ given $S_{t}$

$$
r_{t}=\psi_{0}\left(S_{t}\right)+\psi_{1}\left(S_{t}\right)^{\prime} X_{t}
$$

where $\psi_{0}\left(S_{t-}\right)$ is a scalar and $\psi_{1}\left(S_{t-1}\right)$ is a $L \times 1$ vector.

We further specify the pricing kernel $M_{t}$ as ${ }^{3}$

$$
\begin{aligned}
\frac{d M_{t}}{M_{t-}}= & -r_{t-} d t-\lambda_{D, t}^{\prime} d W_{t}-\lambda_{J, t}^{\prime}\left(d N_{t}-\delta_{J, t} d t\right) \\
& -\int_{E} \lambda_{S}\left(z, X_{t-}\right)\left[\mu(d t, d z)-\gamma_{\mu}(d t, d z)\right]
\end{aligned}
$$

where $\lambda_{D, t} \equiv \lambda_{D}\left(X_{t-}, S_{t-}\right)$ is a $L \times 1$ vector of market prices of diffusion risk; $\lambda_{J, t} \equiv \lambda_{J}\left(X_{t-}, S_{t-}\right)$ is a $L \times 1$ vector of market prices of jump risk conditioning on $X_{t-}$ and $S_{t-}$; and $\lambda_{S}\left(z, X_{t-}\right)$ is the market price of regimeswitching (from regime $i$ to regime $j$ ) risk given $X_{t-}$. Wu and Zeng (2005) provided a general equilibrium interpretation of the regime-switching risk.

\section{$2.2 \quad$ The Term Structure of Interest Rates}

The specifications above complete the model for the term structure of interest rates, which can be solved by a change of probability measure. Specifically, for fixed $T>0$, we define the equivalent martingale measure $\mathbf{Q}$ by the Radon-Nikodym derivative

$$
\frac{d Q}{d P}=\xi_{T} / \xi_{0}
$$

where for $t \in[0, T]$

$$
\begin{aligned}
\xi_{t}= & \left(e^{-\int_{0}^{t} \lambda_{D, u}^{\prime} d W(u)-\frac{1}{2} \int_{0}^{t} \lambda_{D, u}^{\prime} \lambda_{D, u} d u}\right) \times \\
& \left(e^{\int_{0}^{t} \lambda_{J, u}^{\prime} \delta_{J, u} d u+\int_{0}^{t} \log \left(1-\lambda_{J, u}\right)^{\prime} d N_{t}}\right) \times \\
& \left(e^{\int_{0}^{t} \int_{E} \lambda_{S}\left(z, X_{u-}\right) \gamma_{\mu}(d u, d z)+\int_{0}^{t} \int_{E} \log \left(1-\lambda_{S}\left(z, X_{u-}\right)\right) \mu(d u, d z)}\right)
\end{aligned}
$$

In the absence of arbitrage, the price at time $t$ of a default-free pure

\footnotetext{
${ }^{3}$ Absence of arbitrage is sufficient for the existence of the pricing kernel under certain technical conditions, as pointed out by Harrison and Kreps (1979)
} 
discount bond that matures at $T, P(t, T)$, can be obtained as,

$$
P(t, T)=E_{t}^{\mathbf{Q}}\left(e^{-\int_{t}^{T} r_{u} d u}\right)
$$

with the boundary condition $P(T, T)=1$.

Moreover, under probability measure $\mathbf{Q}$,

$$
\begin{aligned}
d X_{t} & =\tilde{\Theta}\left(X_{t-}, S_{t-}\right) d t+\Sigma\left(X_{t-}, S_{t-}\right) d \tilde{W}_{t}+J\left(X_{t-}, S_{t-}\right) d \tilde{N}_{t} \\
d S_{t} & =\int_{E} \zeta(z) \tilde{\mu}(d t, d z)
\end{aligned}
$$

where $\tilde{\Theta}\left(X_{t-}, S_{t-}\right)=\Theta\left(X_{t-}, S_{t-}\right)-\Sigma\left(X_{t-}, S_{t-}\right) \lambda_{D}\left(X_{t-}, S_{t-}\right) ; \tilde{W}_{t}$ is a $L \times 1$ standard Brownian motion under $\mathbf{Q} ; \tilde{N}_{t}$ is a $L \times 1$ vector of Poisson processes with intensity $\tilde{\delta}_{J}\left(X_{t-}, S_{t-}\right)$ whose elements are given by $\tilde{\delta}_{i, J}\left(X_{t-}, S_{t-}\right)=$ $\left[1-\lambda_{i, J}\left(X_{t-}, S_{t-}\right)\right] \delta_{i, J}\left(X_{t-}, S_{t-}\right)$ for $i=1, \cdots, L ; \tilde{\mu}(t, A)$ is the marked point process with intensity matrix $\tilde{H}\left(X_{t-}\right)=\left\{\tilde{h}\left(z, X_{t-}\right)\right\}=\left\{h\left(z, X_{t-}\right)(1-\right.$ $\left.\left.\lambda_{S}\left(z, X_{t-}\right)\right)\right\}$. The compensator of $\tilde{\mu}(t, A)$ under $\mathbf{Q}$ becomes

$$
\tilde{\gamma}_{\mu}(d t, d z)=\left(1-\lambda_{S}\left(z, X_{t-}\right)\right) \gamma_{\mu}(d t, d z)=\tilde{h}\left(z, X_{t-}\right) \mathbf{I}\left\{S_{t-}=i\right\} \epsilon_{z}(d z) d t
$$

Without loss of generality, let $P(t, T)=f\left(t, X_{t}, S_{t}, T\right)$. The following proposition gives the partial differential equation determining the bond price.

Proposition 1 The price of the default-free pure discount bond $f(t, X, S, T)$ defined in (9) satisfies the following partial differential equation

$$
\begin{aligned}
\frac{\partial f}{\partial t} & +\frac{\partial f}{\partial X^{\prime}} \tilde{\Theta}+\frac{1}{2} \operatorname{tr}\left(\frac{\partial^{2} f}{\partial X \partial X^{\prime}} \Sigma \Sigma^{\prime}\right) \\
& +\int_{E} \Delta_{S} f \tilde{h}(z) \mathbf{I}\left\{S_{t-}=i\right\} \epsilon_{z}(d z)+\tilde{\delta}_{J}^{\prime} \overline{\Delta_{X} f}=r f
\end{aligned}
$$

with the boundary condition $f(T, X, S, T)=1$. Where $\Delta_{S} f=f\left(t-, X_{t-}, S_{t-}+\right.$

\footnotetext{
${ }^{4}$ Note that if the market price of jump risk $\lambda_{J, t}$ also depends on the jump size, the conditional density of the jump size $J_{i}\left(X_{t-}, S_{t-}\right)$ under $\mathbf{Q}$ (conditioning on $X_{t-}$ and $S_{t-}$ ) is given by $\tilde{g}\left(J_{i} \mid X_{t-}, S_{t-}\right)=\frac{1-\lambda_{J}\left(X_{t-}, S_{t-}, J_{i}\right)}{1-\bar{\lambda}_{J}\left(X_{t-}, S_{t-}\right)} g\left(J_{i} \mid X_{t-}, S_{t-}\right)$, where $\bar{\lambda}_{J}$ is the conditional mean of the jump size given $X$ and $S$.
} 
$\zeta(z), T)-f\left(t-, X_{t-}, S_{t-}, T\right)$, and

$$
\Delta_{X} f=\left(\begin{array}{c}
f\left(t-, X_{t-}+J_{1}, S_{t-}\right)-f\left(t-, X_{t-}, S_{t-}\right) \\
\vdots \\
f\left(t-, X_{t-}+J_{L}, S_{t-}\right)-f\left(t-, X_{t-}, S_{t-}\right)
\end{array}\right)
$$

and $\overline{\Delta_{X} f}=E_{J}^{\mathbf{Q}}\left(\Delta_{X} f\right)=\int \Delta_{X} f g\left(J \mid X_{t-}, X_{t-}\right) d J$, i.e. the mean of $\Delta_{X} f$ conditioning on $X_{t-}$ and $S_{t-}$ under $\mathbf{Q}$.

\section{An Affine Model with Regime-Dependent Jumps}

In general equation (12) doesn't admit a closed form solution for bond prices. In this section we provide an example of the term structure model that can be solved in analytical form using log-linear approximation. The example falls into the class of affine term structure models. Duffie and Kan (1996) and Dai and Singleton (2000), among other, have detailed discussions of affine term structure models under diffusions. Duffie (2002) introduces a broader class of semi-affine models that have more flexible specifications for the market prices of risk. Duffie, Pan and Singleton (2000) deals with general asset pricing under affine jump-diffusions. Dai, Singleton and Yang (2003), Bansal and Zhou (2002) and Landen (2000) also use affine structure for their regime-switching models. Following this literature, we make the following assumptions:

(1) $\Theta\left(X_{t-}, S_{t-}\right)=\Theta_{0}\left(S_{t-}\right)+\Theta_{1}\left(S_{t-}\right) X_{t-}$ where $\Theta_{0}\left(S_{t-}\right)$ is a $L \times 1$ vector and $\Theta_{1}\left(S_{t-}\right)$ is a $L \times L$ matrix;

(2) $\Sigma\left(X_{t-}, S_{t-}\right)$ is a $L \times L$ diagonal matrix with the $i$ th diagonal element given by $\left[\Sigma\left(X_{t-}, S_{t-}\right)\right]_{i i}=\sqrt{\sigma_{0, i}\left(S_{t-}\right)+\sigma_{1, i}^{\prime}\left(S_{t-}\right) X_{t-}}$ for $i=1, \cdots, L$;

(3) $\delta_{J}\left(X_{t-}, S_{t-}\right)=\delta_{J}\left(S_{t-}\right)$, which is a $L \times 1$ vector;

(4) $\int e^{J^{\prime} B\left(T-t, S_{t-}\right)} g\left(J \mid X_{t-}, S_{t-}\right) d J=G\left(T-t, S_{t-}\right)$ for some $L \times 1$ vector $B\left(T-t, S_{t-}\right)$;

(5) $h\left(z, X_{t-}\right)=e^{h_{0}(z)+h_{1}^{\prime}(z) X_{t-}}$;

(6) $\lambda_{D}\left(X_{t-}, S_{t-}\right)=\Sigma\left(X_{t-}, S_{t-}\right) \lambda_{D}\left(S_{t-}\right)$ for some $L \times 1$ vector $\lambda_{D}\left(S_{t-}\right)$;

(7) $\lambda_{J}\left(X_{t-}, S_{t-}\right)=\lambda_{J}\left(S_{t-}\right)$, which is a $L \times 1$ vector and $\lambda_{J}\left(S_{t-}\right)<1$ for all $S_{t-}$; 
(8) $\lambda_{S}\left(z, X_{t-}\right)=1-e^{\lambda_{0, S}(z)+\lambda_{1, S}^{\prime}(z) X_{t-}}$, where $\lambda_{1, S}(z)$ is $L \times 1$ vector.

Notice that, among the above assumptions, (3) and (4) imply that both the intensity and magnitude of the discreet jump $d N_{t}$ are regime-dependent. This specification can capture the feature of the interest rate movements that jumps of the same direction are persistent. The model can be easily generalized so that the jump intensity also depends on state variable $X_{t-}$. Assumption (5) allows the transition probability of regime shifts to be timevarying. The last three assumptions deal with the market prices of risk. We assume in (6) that the market price of the diffusion risk is proportional to the diffusion term of the state variable $X_{t}$ as in the conventional affine models. A natural extension would be to use the semi-affine specification of Duffie (2002). We assume in (7) and (8) that the market price of the jump risk is a regime-dependent constant and the market price of regime switching risk is an affine function under log-linear approximation.

Under these parameterizations, the state variable $X_{t}$ and the Markov chain $S_{t}$ will preserve the affine structure under the risk-neutral measure $\mathbf{Q}$. In particular, let

$$
\begin{aligned}
& \Sigma_{0}\left(S_{t-}\right)=\left[\sigma_{0,1}\left(S_{t-}\right), \cdots, \sigma_{0, L}\left(S_{t-}\right)\right]^{\prime} \\
& \Sigma_{1}\left(S_{t-}\right)=\left[\sigma_{1,1}\left(S_{t-}\right), \cdots, \sigma_{1, L}\left(S_{t-}\right)\right]
\end{aligned}
$$

and

$\Lambda_{D}\left(S_{t-}\right)=\left(\begin{array}{ccc}\lambda_{D, 1}\left(S_{t-}\right) & & \\ & \ddots & \\ & & \lambda_{D, L}\left(S_{t-}\right)\end{array}\right), \quad \Lambda_{J}\left(S_{t-}\right)=\left(\begin{array}{lll}\lambda_{J, 1}\left(S_{t-}\right) & & \\ & \ddots & \\ & & \lambda_{J, L}\left(S_{t-}\right)\end{array}\right)$

The drift term $\tilde{\Theta}$, the regime switching intensity $\tilde{h}$ and the jump intensity $\tilde{\delta}_{J}$ in (12) of Proposition 1 are given by

$$
\begin{aligned}
\tilde{\Theta}(X, S) & =\tilde{\Theta}_{0}(S)+\tilde{\Theta}_{1}(S) X \\
& =\left[\Theta_{0}(S)-\Lambda_{D}(S) \Sigma_{0}(S)\right]+\left[\Theta_{1}(S)-\Lambda_{D}(S) \Sigma_{1}^{\prime}(S)\right] X \\
\tilde{h}(z, X) & =e^{\tilde{h}_{0}(z)+\tilde{h}_{1}^{\prime}(z) X} \\
& =e^{\left[h_{0}(z)+\lambda_{0, S}(z)\right]+\left[h_{1}(z)+\lambda_{1, S}(z)\right]^{\prime} X}
\end{aligned}
$$

and

$$
\tilde{\delta}_{J}(S)=\left(\mathbf{I}-\Lambda_{J}(S)\right) \delta_{J}(S)
$$


Using a log-linear approximation similar to that in Bansal and Zhou (2002), we can solve for the term structure of interest rates as follows

Proposition 2 Under the assumption (1)-(8) and that $r_{t}=\psi_{0}\left(S_{t}\right)+\psi_{1}\left(S_{t}\right)^{\prime} X_{t}$, the price at time $t$ of a default-free pure discount bond with maturity $\tau$ is given by $P(t, \tau)=e^{A\left(\tau, S_{t}\right)+B\left(\tau, S_{t}\right)^{\prime} X_{t}}$ and the $\tau$-period interest rate is given by $R(t, \tau)=-\frac{A\left(\tau, S_{t}\right)}{\tau}-\frac{B\left(\tau, S_{t}\right)^{\prime} X_{t}}{\tau}$, where $A(\tau, S)$ and $B(\tau, S)$ are determined by the following differential equations

$$
\begin{aligned}
& -\frac{\partial B(\tau, S)}{\partial \tau}+\tilde{\Theta}_{1}(S)^{\prime} B(\tau, S)+\frac{1}{2} \Sigma_{1}(S) B^{2}(\tau, S) \\
& +\int_{E}\left[e^{\Delta_{s} A}\left(\Delta_{s} B+\tilde{h}_{1}(z)\right)-\tilde{h}_{1}(z)\right] e^{\tilde{h}_{0}(z)} \mathbf{1}(S=i) \epsilon_{z}(d z)=\psi_{1}(S)
\end{aligned}
$$

and

$$
\begin{aligned}
& -\frac{\partial A(\tau, S)}{\partial \tau}+\tilde{\Theta}_{0}(S)^{\prime} B(\tau, S)+\frac{1}{2} B(\tau, S)^{\prime} \Sigma_{0}(S) B(\tau, S)+\tilde{\delta}_{J}(S)^{\prime} E_{J}\left[e^{J^{\prime} B(\tau, S)}-\mathbf{1}\right] \\
& +\int_{E}\left[e^{\Delta_{s} A}-1\right] e^{\tilde{h}_{0}(z)} \mathbf{1}(S=i) \epsilon_{z}(d z)=\psi_{0}(S)
\end{aligned}
$$

with boundary conditions $A(0, S)=0$ and $B(0, S)=0$, where $\Delta_{s} A=$ $A(\tau, S+\zeta(z))-A(\tau, S), \Delta_{s} B=B(\tau, S+\zeta(z))-B(\tau, S)$, and $B^{2}(\tau, S)=$ $\left(B_{1}^{2}(\tau, S), \cdots, B_{L}^{2}(\tau, S)\right)^{\prime}$.

Note that Proposition 2 nests many of the existing regime-switching models of the term structure of interest rates. For example, neither Landen (2000), Bansal and Zhou (2002) nor Dai, Singleton and Yang (2003) include a jump component, therefore $E_{J}\left[e^{J^{\prime} B(\tau, s)}-1\right]=0$. Both Landen $(2000)$ and Bansal and Zhou (2002) assume constant transition probabilities for the Markov process, hence $h_{1}(z)=\tilde{h}_{1}(z)=0$. Dai, Singleton and Yang (2003) allows for time-varying transition probabilities under the physical measure. But in order to obtain exact analytical solution, they impose that the transition probabilities is constant under the risk-neutral measure, $\tilde{h}_{1}(z)=0$, and assume Gaussian distribution for state variable $X_{t}$, therefore $\Delta_{s} B=0 .{ }^{5}$ The latter specification is also shared by Landen (2000). Moreover, while Landen (2000) is silent on the market price of risk, Bansal and Zhou (2002) assumes that the risk of regime shifts is not priced, i.e. $\tilde{h}_{0}(z)=h_{0}(z)$. The

\footnotetext{
${ }^{5}$ Under these assumptions, (16) and (17) yield the exact solution for the term structure of interest rates.
} 
model proposed in the present paper relaxes those restrictions in a tractable way, and it can be estimated by simulation-based method such as Efficient Method of Moments.

\section{References}

[1] Ahn, C and H. Thompson (1988) "Jump Diffusion Processes and the Term Structure of Interest Rates", Journal of Finance 43, 155-174.

[2] Bansal, R. and H. Zhou (2002) "Term Structure of Interest Rates with Regime Shifts", forthcoming, Journal of Finance.

[3] Das, S. (2002) "The Surprise Element: Jumps in Interest Rates", Journal of Econometrics 106, 27-65.

[4] Dai, Q. and K. Singleton (2000) "Specification Analysis of Affine Term Structure Models", Journal of Finance, vol. LV, 1943-78.

[5] Dai, Q., K. Singleton and W. Yang (2003) "Regime Shifts in Dynamic Term Structure Model of the U.S. Treasury Bond Yields", working paper, Stanford University.

[6] Duffie, D. and R. Kan (1996) "A Yield-Factor Model of Interest Rates", Mathematical Finance 6, 379-406.

[7] Duffie, D., J, Pan and K. Singleton (2000) "Transform Analysis and Asset Pricing for Affine Jump-Diffusions", Econometrica 68, 1343-1376.

[8] Duffie, G. (2002) "Term Premia and Interest Rate Forecasts in Affine Models" Journal of Finance, 57, 405-443.

[9] Harrison, M. and D. Kreps (1979) "Martingales and Arbitrage in Multiperiod Security Markets", Journal of Economic Theory 20, 381-408.

[10] Landen, C. (2000) "Bond Pricing in a Hidden Markov Modle of the Short Rate", Finance and Stochastics 4, 371-389.

[11] Last, G. and A. Brandt (1995) Marked Point Processes on the Real Line, Springer, New York.

[12] Piazzesi, M. (2005) "Bond Yields and the Federal Reserve", Journal of Political Economy 113, 311-344. 
[13] Wu, S. and Y. Zeng (2005) "A General Equilibrium Model of the Term Structure of Interest Rates under Regime-siwthcing Risk", International Journal of Theortical and Applied Finance, forthcoming. 